Document downloaded from:

http://hdl.handle.net/10251/79149

This paper must be cited as:

Brimont, ACJ.; Hu, X.; Cueff, S.; Rojo-Romeo, P.; Saint Girons, G.; Griol Barres, A.; Zanzi, A.... (2016). Low-Loss and Compact Silicon Rib Waveguide Bends. IEEE Photonics Technology Letters. 28(3):299-302. doi:10.1109/LPT.2015.2495230.

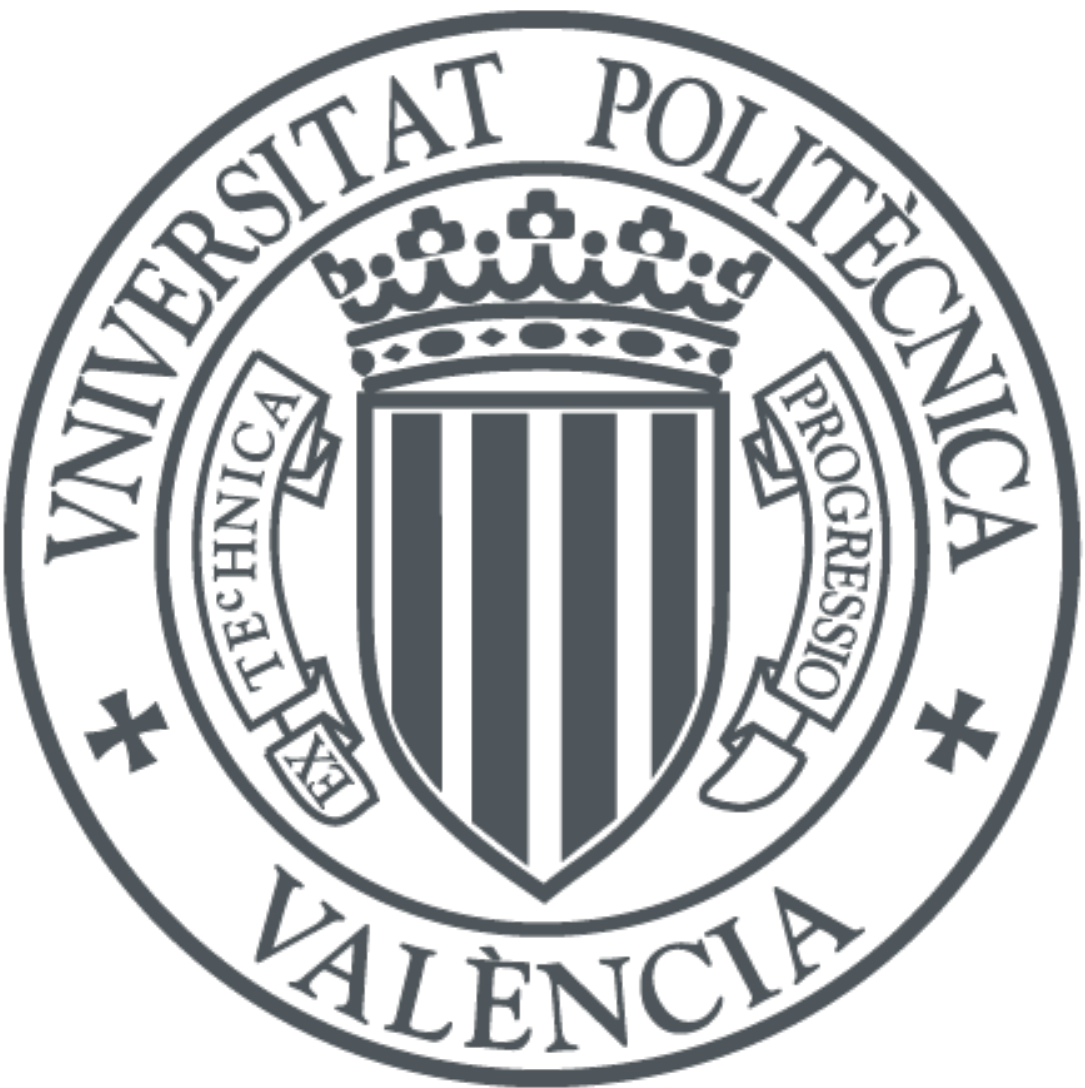

The final publication is available at

http://dx.doi.org/10.1109/LPT.2015.2495230

Copyright Institute of Electrical and Electronics Engineers (IEEE)

Additional Information 


\title{
Low-loss and compact silicon rib waveguide bends
}

\author{
Antoine Brimont, Xuan Hu, Sébastien Cueff, Pedro Rojo Romeo, Guillaume Saint Girons, Amadeu \\ Griol, Andrea Zanzi, Pablo Sanchis, Régis Orobtchouk ${ }^{*}$
}

\begin{abstract}
Waveguide bends support intrinsically leaky propagation modes due to unavoidable radiation losses. It is known that the losses of deep-etched/strip waveguide bends increase inevitably for decreasing radius. Here, we theoretically and experimentally demonstrate that this result is not directly applicable to shallow-etched/rib waveguide bends. Indeed, we show that the total losses caused by the bends reach a local minimum value for a certain range of compact radii and rib waveguide dimensions. Specifically, we predicted minimum intrinsic losses $<0.1 \mathrm{~dB} / 90^{\circ}$ turn within the range of $25-30 \mu \mathrm{m}$ bend radii in a $220 \mathrm{~nm}$-thick and400nm-wide silicon rib waveguide with $70 \mathrm{~nm}$ etching depth. This unexpected outcome, confirmed by experimental evidence, is due to the opposite evolution of radiation (bending) losses and losses caused by the coupling to lateral slab modes (slab leakage) as a function of the bend radius, hence creating an optimum loss region. This result may have important implications for the design of compact and low-loss silicon nanophotonic devices.
\end{abstract}

Index Terms-Integrated optics, silicon photonics, rib waveguides, waveguide bends, optical design.

\section{INTRODUCTION}

L OW-loss silicon waveguides, allowing efficient on-chip dissemination of optical signals are the backbone of silicon-based nanophotonics. Among the existing geometries, rib waveguide architectures are usually chosen to leverage industrial fabrication of advanced silicon based telecom and datacom photonic integrated circuits (PICs) [1-2]. The reason for this is that passive and active photonic components can readily be patterned with a single shallow-etch step using current complementary-metal-oxide-semiconductor (CMOS) fabrication techniques. Indeed, a rib waveguide-based interconnect layer decreases the complexity of the process by ruling out potential fabrication dependent errors such as alignment between strip/rib Si-photonic layers as well as the extra-cost associated with additional lithography masks.

Typical rib waveguides consist of straight and bend sections exhibiting three sources of losses:

Manuscript received XXX; revised XXX; accepted XXX. This work was supported in part by the European STREP program "FP7-ICT-2013-11619456-SITOGA and "FP7-ICT-2012-10-318240 PhoxTroT". Financial support from TEC2012-38540 LEOMIS is also acknowledged.

A. Brimont, A. Griol, A. Zanzi and P. Sanchis are with the Nanophotonics Technology Center, Universitat Politècnica de Valencia, Camino de Vera s/n 46022 Valencia, Spain (e-mail: abrimont@ntc.upv.es; agriol@ntc.upv.es; anzan@ntc.upv.es; pabsanki@dcom.upv.es).
A) Propagation losses due to sidewall roughness and other process/material dependent effects;

B) Slab leakage losses, produced by coupling to the lateral slab and radius dependent;

C) Bending losses which are inherent to the bend and radius dependent.

This is in contrast to strip (deep-etched) waveguides which only suffer from two sources of losses, namely A (propagation losses caused by sidewall roughness) and C (bending losses).

The aim of this paper is to demonstrate that the singular refractive index distribution associated with the presence of the slab in rib waveguides mitigates the bending losses in a certain range of bend radii.

Although straight rib waveguide parameters are always chosen to rule out slab leakage losses (B) by confining most of the modal power in the ridge area, aggressive bending may cause the confined light to leak into the slab. Additionally, bending losses (C) rapidly increase as the bend radius becomes smaller. Therefore, to prevent on-chip-losses from becoming prohibitive, radii of about hundreds of microns are usually chosen to achieve low-loss in rib waveguide bends [3]. As a result, the footprint of integrated photonic devices featuring these large radii rib waveguide bends may reach unacceptable proportions for large scale PICs.

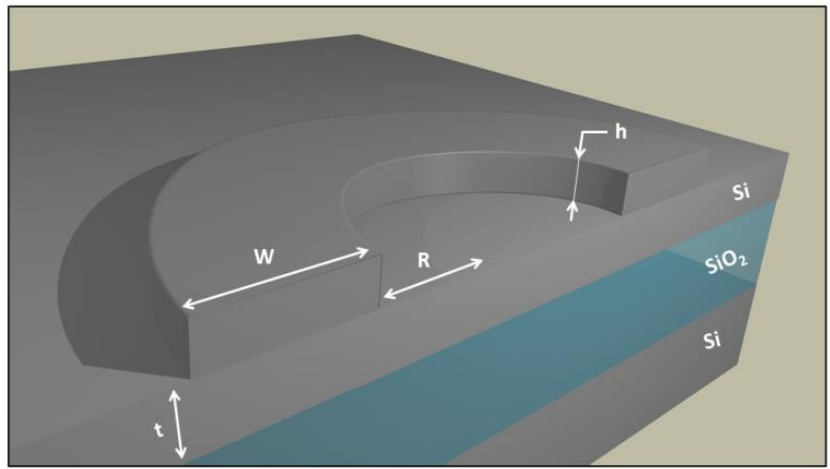

Fig. 1. Geometry of the curved rib waveguide ( $\mathrm{W}=0.4 \mu \mathrm{m}, \mathrm{h}=0.07 \mu \mathrm{m}$ and $\mathrm{t}=0.15 \mu \mathrm{m})$.

X. Hu, S. Cueff, P. Rojo-Romeo, G. Saint-Girons and R. Orobtchouk are with the Institut des Nanotechnologies de Lyon (INL), CNRS UMR5270, 7 avenue Jean Capelle, INSA-Lyon, Villeurbanne 69621, France (e-mail: xuan.hu@insa-lyon.fr; sebastien.cueff@ec-lyon.fr; pedro.rojo-romeo@eclyon.fr; guillaume.saint-girons@ec-lyon.fr; regis.orobtchouk@insa-lyon.fr). 
It has been reported that losses can be mitigated by increasing the etching depth in the outer side of the curvature [4]. However, such a solution requires extra dedicated technological steps, which eventually increases the complexity and cost of the whole fabrication process.

In this paper, we theoretically and experimentally demonstrate that low loss rib waveguide bends can be obtained for a specific range of bend radii without adding more complexity to standard waveguide fabrication processes.

In the following, we present a theoretical analysis confirmed by experimental evidence. We show that losses lower than 0.1 $\mathrm{dB} / 90^{\circ}$ turn (excluding propagation losses due to sidewall roughness and/or process/material dependent effects) can be obtained for a range of bend radius as small as $25 \mu \mathrm{m}$ to $30 \mu \mathrm{m}$.

\section{THEORY AND MODELING}

Modeling of the bends is performed with a home-made finite difference full-vectorial mode solver in cylindrical coordinates [5]. Transparent boundaries conditions are used to accurately calculate the leakage and bending losses introduced by the curvature [6]. Importantly, note that the following calculations have been performed excluding the propagation losses due to sidewall roughness or other process/material dependent effects.

The geometry of the rib waveguide and refractive indices used are depicted on Fig. 1. Width, $W$, thickness of Si layer, $t$, and height, $h$, of the rib waveguide are respectively $0.4,0.15$ and $0.07 \mu \mathrm{m}$. The refractive indices of $\mathrm{Si}$ and $\mathrm{SiO}_{2}$ are set to 3.4758 and 1.4442 at $\lambda=1.55 \mu \mathrm{m}$. Calculated variations of the losses (leakage + bending) of the quasi-TE fundamental mode versus the bend radius is plotted in Fig. 2.

Interestingly, we observe two regimes of low-loss $(<0.1 \mathrm{~dB})$ : one for large bend radius ( $r>200 \mu \mathrm{m})$, and a second one for small radius of about $25 \mu \mathrm{m}$. This atypical behavior implies that one can design waveguide bends with both a small radius and low losses.

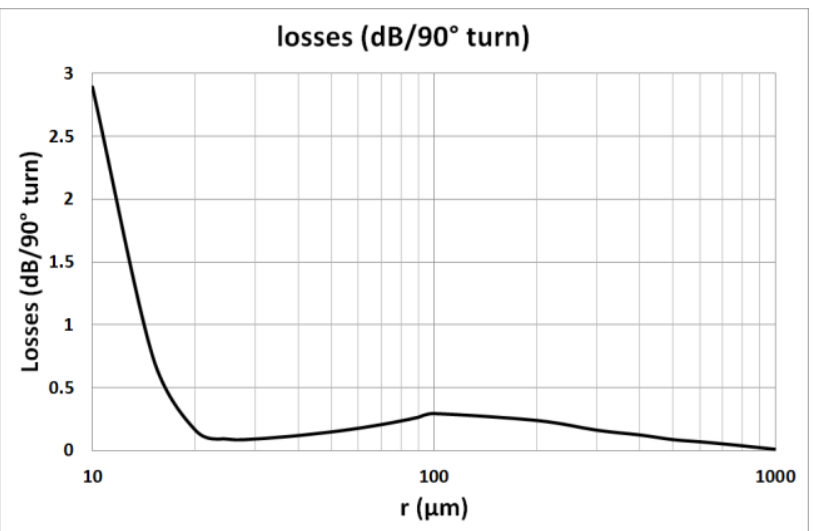

Fig. 2. Evolution of the radiation losses $\left(\mathrm{dB} / 90^{\circ}\right.$ turn $)$ versus the radius $(\mu \mathrm{m})$ for the quasi-TE fundamental mode at $\lambda=1.55 \mu \mathrm{m}$.

As shown in fig. 3, the regime of low-loss reduction for small radius occurs similarly for a wide range of waveguide widths and heights. Interestingly, the minimum loss value $(0.09$ $\mathrm{dB} / 90^{\circ}$ turn) for a radius of about $25 \mu \mathrm{m}$ obtained for the target waveguide geometry $(70 \mathrm{~nm}$ etch and $400 \mathrm{~nm}$ waveguide width) is a local minimum among the parameter scan range.
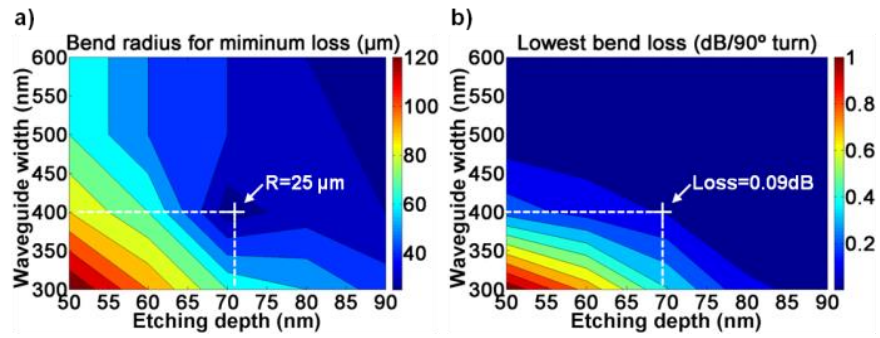

Fig. 3 a) Evolution of the bend radius for minimum loss and b) lowest bend loss versus waveguide width (w) an height (h)

Radiation losses of a rib waveguide bend can be explained via the well-known conformal mapping transformation, in which the wave propagation equation in cylindrical coordinates is approximated by that of a straight waveguide in Cartesian coordinates [7-8]. The results of this conformal transformation induce modifications in the refractive indices of the straight rib waveguide, as reported in Fig. 4. a). As the refractive indices of the materials increase with $r$, there is a threshold value $r_{3}$ above which the effective index $n_{\text {eff }}$ of the propagating mode becomes lower than the refractive index of the cladding. In that case, the evanescent part of the mode becomes propagative and introduces bending losses, as illustrated by the green arrow in Fig. 4. b). One can notice that when the bend radius decreases, the exponential variation of the refractive index with $r$ implies a faster reduction for the threshold value $r_{3}$ than $r_{2}$, hence inexorably increasing bending losses in $\mathrm{dB} / \mathrm{unit}$ length, just as what happens in strip waveguide bends.

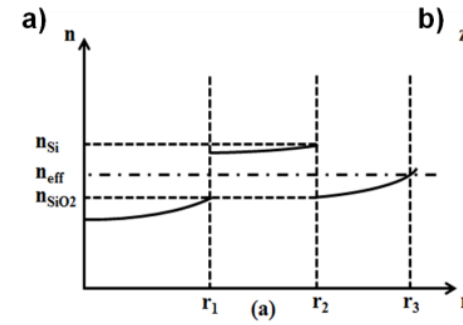

b)

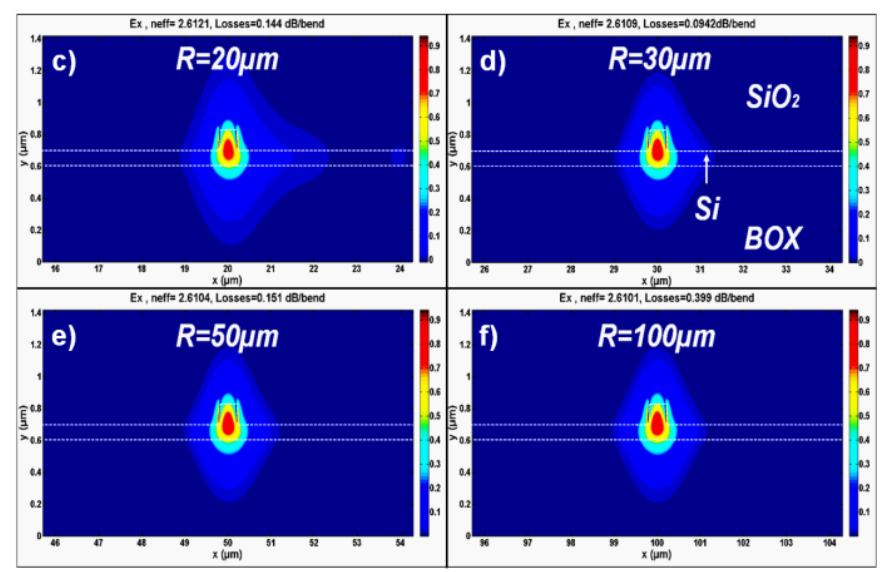

Fig. 4. a) Equivalent refractive index profile (n) of a rib waveguide bend versus radius ( $r$ ) and b) schematic representation of the sources of losses in a rib bend waveguide. Namely, bending losses (Green arrow pointing towards the outer part of the bend, higher $r$ values) and leakage losses produced by coupling to the slab mode (yellow arrows). Mode profiles for c) 20, d) 30 e) 50 and f) 100 $\mu \mathrm{m}$ radii. Respective theoretical losses are $1.44,0.0942,0.151$ and $0.299 \mathrm{~dB} / 90^{\circ}$ bend. Modal asymmetries can be observed for more compact bend radii ( 20 and $30 \mu \mathrm{m})$.

However, it turns out that, in contrast to strip geometries, rib waveguide bends exhibit an additional source of losses 
produced by the coupling of the mode to the lateral Si slab (yellow arrows in Fig. 4 b)). This effect has been used for the rejection of higher order modes in straight rib waveguides [911]. In the case of a rib waveguide bend, the lateral coupling to the slab is not symmetric: in the inner part of the bend, the refractive index of the slab decreases, while in the outer part of the bend the refractive index of the slab increases.

The asymmetry of the refractive index causes the maximum field value of the mode profile to move towards the inner part of the bend (towards the left side, Fig 4a)), therefore reducing the radiation (bending losses) produced by the $r_{3}$ threshold value. Moreover, the same asymmetry shifts the mode upwards, hence contributing to further decrease losses by reducing the coupling to the slab (leakage losses). This shift was also observed by D. Dai and all [12] for high order modes without any physical interpretation. Pictures of the simulated mode profiles in bends with radii of $20,30,50$ and $100 \mu \mathrm{m}$ are respectively shown in Fig. 3. c), d), e) and f).

Therefore, in contrast to their strip counterparts, rib waveguide bends exhibit additional internal loss compensation mechanisms. Furthermore, for a $90^{\circ}$ turn, decreasing the radius of curvature also implies shortening the total length (by a factor $\pi \mathrm{r} / 2$ ) which reduces the total losses per bend due to the shortened arc length. According to our numerical modeling, in the range of curvature between $25 \mu \mathrm{m}$ and $100 \mu \mathrm{m}$, all of the above mentioned effects concur to reduce the total net loss when the curvature is decreased.

\section{EXPERIMENT}

To confirm our theoretical results, we measured the bend losses using the photonic test structures pictured in Fig.5, for different bend radii $(20,30,50$ and $100 \mu \mathrm{m})$. In order to accurately measure only bending (B) and leakage loss (C) contributions within the bend losses, the input waveguide is split into two arms with identical lengths of N. $\pi . R / 2$. The upper output arm contains the bends and its output power is normalized by that of the lower arm, in order to eliminate the propagation losses produced by sidewall roughness. This is to match our theoretical modeling which does not include the losses due to sidewall roughness and process/material dependent issues. Details on the measurement method are given in [13].

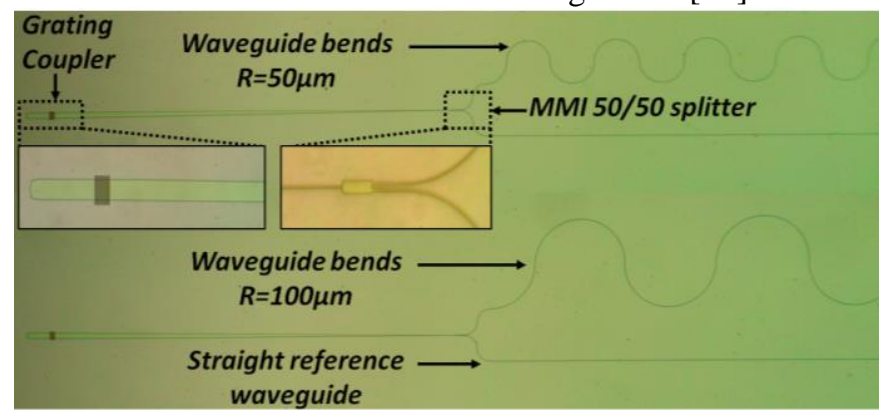

Fig. 5 Silicon photonic test structures used for loss measurement of the rib waveguide bends.

The structures have been fabricated on standard Silicon-oninsulator (SOI) samples with a top $220 \mathrm{~nm}$ thick silicon layer and a buried oxide layer (BOX) thickness of $2 \mu \mathrm{m}$. Silicon waveguides were fabricated with electron beam lithography (RAITH 150) and inductively coupled plasma-reactive ion etching (ICP-RIE). The etch depth is $70 \mathrm{~nm}$ for both waveguides and grating couplers, leaving a $150 \mathrm{~nm}$ thick slab. The shallow-etched passive silicon chip is then covered with $700 \mathrm{~nm}$ silicon oxide using plasma-enhanced chemical vapor deposition (PECVD) at $400^{\circ} \mathrm{C}$ (Centura P5200). Experiments were performed using a set of optical components composed of $\mathrm{N}=0,16$ or $3290^{\circ}$-turn bends.
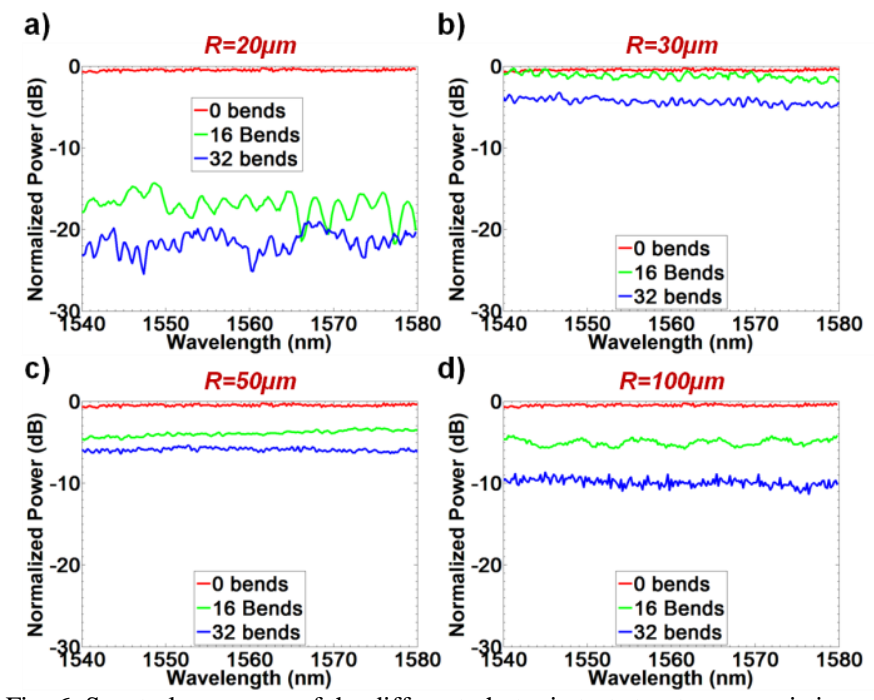

Fig. 6. Spectral responses of the different photonic test structures consisting of 0,16 and $3290^{\circ}$ bends with respective radii of a) 20, b) 30 , c) 50 and b) 100 $\mu \mathrm{m}$.

Spectral responses of the 12 (3 test structures per bend radius) optical test structures are given in Fig. 6 for curvature radii of $20,30,50$ and $100 \mu \mathrm{m}$. In order to extract the loss per $90^{\circ}$ bend, a least-square linear regression versus the number of $90^{\circ}$ turns is performed wavelength by wavelength using a tunable laser (SANTEC TSL 210-F). Linear regression also gives the accuracy of the measurements. The evolution of the losses versus wavelength is plotted on Fig. 7. The bend losses are approximately constant in the range of wavelength between 1540 to $1580 \mathrm{~nm}$ and equal to $0.343 \pm 0.154,0.103 \pm 0.044$, $0.157 \pm 0.0098$, and $0.295 \pm 0.0021 \mathrm{~dB} / 90^{\circ}$ turn at $1.55 \mu \mathrm{m}$ for curvature radii of $20,30,50$ and $100 \mu \mathrm{m}$, respectively. Fig. 8 shows the evolution of the loss per bend in a range of bend radii between 15 and $100 \mu \mathrm{m}$. There is a very good agreement between theory and measurement, without any fit parameters. This is a clear demonstration of a low-loss regime for small radius rib-waveguide bends.

We can notice that the measurement errors increase as the radius decreases. This may be explained by the mode profile mismatch at the transition between straight and bent waveguides. Indeed, the differences in mode shape and effective indices induce additional losses, back reflections and interferences and hence produce oscillations in the spectral response of the device (see Fig. 6 a)).

The deviation observed for a bend radius of $20 \mu \mathrm{m}$ was expected and can be explained as follows: we have considered in our modeling a perfectly smooth waveguide (i.e. no sidewall roughness) experiencing only the losses produced inherently by the bending and the coupling to the slab mode. Yet again, in our measurements, we have ruled out the propagation losses produced by the sidewall roughness and other process/material dependent effect by normalizing the output spectrum of rib waveguide bends by a straight rib waveguide with the same 
length. This normalization has been performed assuming the sidewall roughness similarly affects the losses of both straight waveguide and waveguide bends. Our assumption is confirmed experimentally for bend radii ranging from $30 \mu \mathrm{m}$ to $100 \mu \mathrm{m}$ since a very good agreement between theoretical data and experimental results can be observed.

However, such a normalization assumes the modal distortion produced by the bending is small enough so that the optical modes associated with both geometries interact similarly with the sidewall roughness.
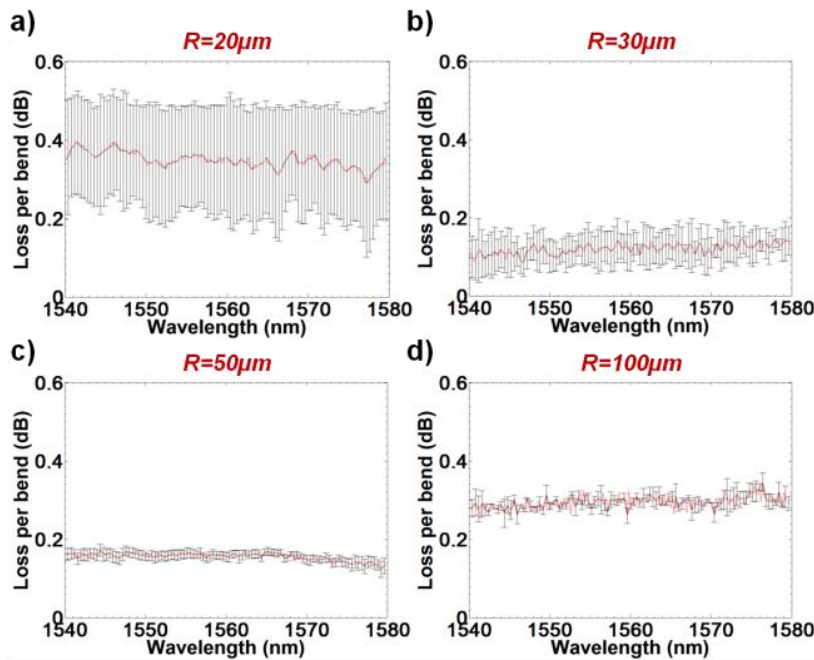

Fig. 7. Evolution of the bend losses versus wavelength for radii of a) 20 , b) 30 , c) 50 and d) $100 \mu \mathrm{m}$.

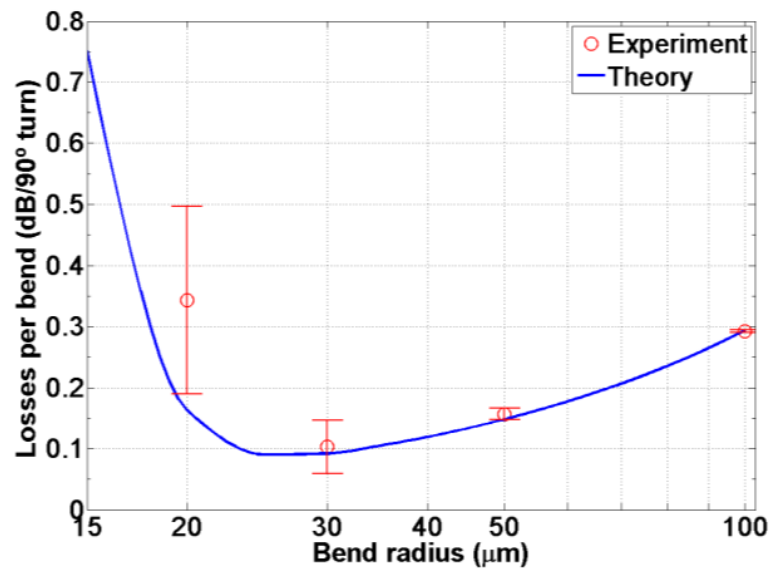

Fig. 8.Evolution of the loss per bend versus the bend radius at $1.55 \mu \mathrm{m}$ wavelength.

Here, for smaller radii $(<20 \mu \mathrm{m})$, the modal distortion resulting from this more aggressive bending increases the interaction of the high field intensity region with the sidewall roughness eventually causing the model to become less accurate. In other words, the spectral normalization of the bent waveguide output by the response of a straight waveguide is less accurate in that range.

In conclusion, we have demonstrated that an optimal value of the bend radius for rib waveguides, allowing low losses and small footprint, can be obtained for the fundamental quasi-TE mode in a wide range of waveguide widths and heights. A physical interpretation based on the evolution of the net losses versus radius, as well as the competition between radiation losses and losses caused by coupling to the lateral slab has been given. These results offer a simple solution to design compact and low-loss nanophotonic silicon rib waveguide bends without altering the fabrication process.

\section{ACKNOWLEDGMENT}

We thank NTC-UPVLC's clean room staff, T. Ivanova, J. Hurtado, L. Bellières, N. Sánchez and R. Bueno for the fabrication of the samples.

\section{REFERENCES}

[1] F.Boeuf, S. Crémer, N. Vulliet, T. Pinguet, A. Mekis, G. Masini, L. Verslegers, P. Sun, A. Ayazi, N.-K.Hon, S. Sahni, Y.Chi, B. Orlando, D. Ristoiu, A. Farcy, F.Leverd, L. Broussous, D. Pelissier-Tanon, C.Richard, L.Pinzelli, R.Beneyton, O.Gourhant, E.Gourvest, Y.Le-Friec, D.Monnier, P.Brun, M.Guillermet, D.Benoit, K.Haxaire, J.R Manouvrier, S. Jan, H. Petiton, J.F. Carpentier, T. Quémerais, C. Durand, D.Gloria, M. Fourel, F. Battegay, Y. Sanchez, E. Batail, F. Baron, P. Delpech, L. Salager, P. De Dobbelaere, B. Sautreuil, "A Multi wavelength 3D-compatible Silicon Photonics Platform on $300 \mathrm{~mm}$ SOI wafers for $25 \mathrm{~Gb} / \mathrm{s}$ Applications," in IEEE Conference on Electron Devices Meeting IEDM, 2013, pp. 13.3.1 - 13.3.4.

[2] S. Sahni, A. Ayazi, C. Yuemeng, A. Dahl, P. De Dobbelaere, S. Gloeckner, H. Kam-Yan, S. Hovey, L. Yi, M. Mack, G. Masini, A. Mekis, M. Peterson, T. Pinguet, J. Schramm, M. Sharp, S. Peng, R. Timpe, L. Verslegers, "Approaches to opto-electronic integration in optical interconnect applications based on Silicon Photonics," in Group IV Photonics (GFP), 2013, pp. 81-82.

[3] G. T. Nguyen, S. R. Tummidi, T. L. Koch, A. Mitchell, "Lateral leakage of TM-like mode in thin-ridge Silicon-on-Insulator bent waveguides and ring resonators," Optics Express, 18(7), 2010, pp. 7243-7259.

[4] K Solehmainen, T Aalto, J Dekker, M Kapulainen, M Harjanne, P Heimala, "Development of multi-step processing in silicon-on-insulator for optical waveguide applications," J. Opt. A: Pure Appl. Opt. 8(7), S455-S460, 2006.

[5] M. Masi, R. Orobtchouk, G. F. Fan, L. Pavesi, "Towards a Realistic Modelling of Ultra-Compact Racetrack Resonators," J. Lightwave Technol. 28(22), 3233-3242, 2010.

[6] G. R. Hadley, "Transparent boundary condition for the beam propagation method," IEEE J. Quant. Electron. 28(1), 363-370, 1992.

[7] M. Heiblum, J. H. Harris, "Analysis of curved optical waveguides by conformal transformation", IEEE J. Quant. Electron. 11(2), 75-85, 1975.

[8] W. Berglud, A. Gopinath, "WKB analysis of bend losses in optical waveguides," J. Lightwave Technol. 18(8), 1161-1166, 2000.

[9] S. P. Pogossian, L. Vescan, and A. Vonsovici, "The single-mode condition for semiconductor rib waveguides with large cross section," $J$. Lightwave Technol. 16(10), 1851-1853, 1998.

[10] R. Orobtchouk, A. Koster, D. Pascal, S. Laval, "Quasi-TE00 single-mode optical waveguide for electro-optical modulation at $1.3 \mu \mathrm{m}$ using standard SIMOX material," in IEEE Proceedings Optoelectronics 144(2), 83-86, 1997.

[11] X. Hu, S. Cueff, P. Rojo Romeo, R. Orobtchouk, "Modeling the anisotropic electro-optic interaction in hybrid silicon-ferroelectric optical modulator," Opt. Express 23(2), 1699-1714, 2015.

[12] D. Dai, S. He, "Analysis of characteristics of bent rib waveguides," $J$. Opt. Soc. Am. A 21(1), 113-121, 2004.

[13] G. Fan, R. Orobtchouk, B. Han, J.-M. Fedeli, X. Liu, and Z. Zhen, "The Basic Building Block of -Router With Multimode-Interference Waveguide Crossings on Silicon-on-Insulator Technology," J. Lightwave Technol. 30(16), 2633-2636, 2012. 